\title{
OPERACIONALIZAÇÃO DE ESCALA PARA ANÁLISE DE PADRÃO DE MEDIAÇÃO MATERNA: UM ESTUDO COM DÍADES MÃE-CRIANÇA COM DEFICIÊNCIA VISUAL
}

\author{
SCALE OPERATIONALIZATION FOR ANALYSIS OF MATERNAL MEDIATION
}

PATTERNS: A STUDY WITH MOTHER - CHILD WITH VISUAL IMPAIRMENT DYADS

\author{
Ana Cristina Barros da CUNHA ${ }^{1}$ \\ Sônia Regina Fiorim ENUMO² \\ Claudia Patrocínio Pedroza CANAL ${ }^{3}$
}

\begin{abstract}
RESUMO: o desenvolvimento cognitivo da criança com necessidades especiais pode ser afetado por padrões inadequados de mediação materna, decorrente das baixas expectativas das mães acerca da capacidade cognitiva infantil, que se constroem a partir da compreensão inadequada acerca dos reais limites e possibilidades de aprendizagem da criança. Nesse sentido, faz-se relevante a avaliação do padrão de mediação materna por instrumentos que forneçam indicadores de análise da mediação presente na interação mãe-criança. Com esse objetivo foi operacionalizada a Mediated Learning Experience (MLE) Rate Scale (Escala de Avaliação de Experiência de Aprendizagem Mediada - Escala MLE), elaborada por Carol Lidz em 1991, com base na Teoria da Experiência de Aprendizagem Mediada. É uma proposta de avaliação da interação adulto-criança, na qual são avaliados os comportamentos mediadores do adulto. Esses comportamentos podem favorecer a construção de expectativas mais otimistas e de um padrão de mediação mais adequado de interação. Após a tradução da Escala MLE, elaboraram-se 36 categorias comportamentais para análise do padrão de mediação materna, organizadas em 4 níveis, para cada um dos 12 componentes da Escala: intencionalidade, significação, transcendência, atenção partilhada, experiência partilhada, regulação na tarefa, elogiar, desafio, diferenciação psicológica, responsividade contingente, envolvimento afetivo. Esta versão adaptada foi aplicada em 12 díades mãe-criança com deficiência visual (5-9 anos), filmadas em 3 sessões de jogos de dominó, com níveis crescentes de dificuldade. A versão operacionalizada da Escala MLE mostrou-se ferramenta útil à análise quantitativa e qualitativa da interação adulto-criança, em situação semi-estruturada de aprendizagem, corroborado por índice de concordância aceitável $(77,2 \%)$.
\end{abstract}

PALAVRAS-CHAVE: interação mãe-criança; avaliação da mediação; mediated learning experience (mle) rate scale; crianças com deficiência visual; desenvolvimento cognitivo; educação especial.

\begin{abstract}
: the cognitive development of children with special needs can be affected by inadequate maternal mediation patterns, due to the mother's low expectations of her child's cognitive performance, which are affected by her inadequate comprehension of her child's actual learning limitations and possibilities. Thus, it is essential to asses the mother's mediation patterns using instruments that will result in indicators enabling the analysis of mediation occurring during mother child interaction. Toward this aim, the Mediated Learning Experience (MLE) Rate Scale, elaborated by Carol Lidz in 1991, based on the Mediated Learning Experience Theory was adapted and applied. This instrument assesses adult-child interaction, focusing on the adult's mediation behaviors. These behaviors can promote the building of higher expectations as well as a more appropriate mediation pattern of interaction. After translating the MLE Scale into Portuguese, 36 behavioral categories were created to analyze the mother mediation pattern in 4 levels for each of the 12 components of the MLE Scale: intentionality, meaning, transcendence, joint regard, sharing of experiences, task regulation, encouragement, challenge, psychological differentiation, contingent responsiveness, affective involvement. This adapted version was applied to 12 mother-visually impairment children (5-9 years of age) dyads; 3 domino games sessions with increasing levels of difficulty were videotaped. The results showed that the adaptation of MLE Scale was useful in the quantitative and qualitative analysis the adult-child interactions in semistructured learning situations, corroborated by an acceptable agreement rate $(77.2 \%)$.
\end{abstract}

KEYWORDS: mother-child interaction; mediation assessment; mediated experience learning (mle) rate scale; visually impaired children; cognitive development; special education.

${ }^{1}$ Doutora, URFJ - Instituto de Psicologia, Departamento de Psicologia Clínica - acbcunha@yahoo.com.br 2 - Doutora, UfES - Programa de Pós-Graduação em Psicologia, bolsista do CNPq sonia.enumo@pesquisador.cnpq.br

${ }^{3}$ Doutoranda, UFES - Pós-Graduação em Psicologia, bolsista da CAPES - claudiapedroza@uol.com.br 


\section{INTRODUÇÃO}

A interação familiar pode ser compreendida como um suporte importante para promoção de todas as áreas do desenvolvimento infantil. Mais especificamente para o desenvolvimento cognitivo, a família assume papel fundamental, uma vez que os pais, ao desempenhar a função de mediadores no processo de aprendizagem do filho, promovem situações que favorecem as funções cognitivas e, conseqüentemente, otimizam os potenciais cognitivos da criança (TZURIEL, 1999, 2001).

Nesse contexto, as mães são especialmente importantes, pois, na maioria das vezes, são elas que interagem com a criança na maior parte do tempo, tendo maior controle sobre os reforçadores do comportamento da criança que são mais poderosos. Igualmente, na interação com a criança, as mães podem ser treinadas para assumir um papel mediador que priorize a aplicação de estratégias de mediação do desenvolvimento infantil (LINHARES; MARTINS; KLEIN, 2004; TZURIEL, 1999, 2001).

Segundo esses autores, no contexto de uma interação mãe-criança mediada, a criança adquire capacidade de resolver problemas de forma mais segura e ordenada, solicitando ajuda do outro quando necessita e usando essa ajuda apropriadamente, além de generalizar essa aprendizagem para outros contextos, como, por exemplo, o ambiente escolar, especialmente quando a criança tem necessidades educativas especiais (NEE).

A criança com necessidades educativas especiais (NEE), como a deficiência visual (DV), por exemplo, deve ser exposta, sistematicamente e de forma planejada, a situações de aprendizagem que a criança normal vivencia de modo assistemático e não planejado. Tais situações de interação a serem oferecidas à criança com DV devem ter como princípio uma mediação de qualidade que promova o desenvolvimento infantil como um todo (BATISTA; ENUMO, 2000; CUNHA; ENUMO; PEDROZA, 2004; FONSECA; CUNHA, 2003).

Entretanto, cabe questionar como pode ser prevista a quantidade e a qualidade de situações de experiência de aprendizagem mediada (EAM) necessárias para promover o pleno potencial de aprendizagem da criança? E como intervir na interação da mãe com a criança, a fim de cumprir esse objetivo?

Considerando essas questões, o presente estudo apresenta uma proposta de operacionalização da Mediated Learning Experience Rate Scale - MLE Scale - Escala de Avaliação da Experiência de Aprendizagem Mediada (LIDZ, 1991), que se apresenta como adequada à avaliação do nível de EAM na interação de qualquer adulto-mediador com a criança (ZAMBRANA-ORTIZ; LIDZ, 1995). 
INTERAÇÃO MÃE-CRIANÇA COMO SUPORTE PARA PROMOÇÃO DO DESENVOLVIMENTO COGNITIVO INFANTIL SOB A PERSPECTIVA DA TEORIA DA EXPERIÊNCIA DE APRENDIZAGEM MEdiada (EAM)

Existem duas formas básicas da criança se desenvolver cognitivamente na interação com o ambiente: (a) através da aprendizagem por exposição direta aos estímulos (fontes de informação), isto é, do contato direto com os acontecimentos e as situações; (b) por meio da experiência de aprendizagem mediada (EAM), ou seja, o processo de aprendizagem que ocorre quando uma outra pessoa serve como um intérprete do estímulo ambiental, tornando esse estímulo relevante e significativo para a criança (FEUERSTEIN; FEUERSTEIN, 1991; FONSECA, 2002; FONSECA; CUNHA, 2003; KLEIN, 1996; LIDZ, 1991, 2000, 2002; TZURIEL, 1999, 2001).

Embora o desenvolvimento cognitivo resulte dessas duas modalidades de interação entre o indivíduo e o ambiente, é a experiência de aprendizagem mediada (EAM), única e exclusiva da espécie humana, que afeta sensivelmente o desenvolvimento da estrutura cognitiva infantil, favorecendo, assim, a modificabilidade cognitiva (cognitive modifiability) estrutural da criança (TZURIEL, 1999, 2001).

Quando a criança se depara sozinha com as regras e princípios da natureza, ela pode não se beneficiar da sabedoria acumulada de sua cultura, sendo, então, a intervenção de uma outra pessoa necessária. No caso da interação mãe-criança, a EAM ocorre quando a mãe auxilia a criança a interpretar suas experiências de vida e a adquirir dessas experiências regras e princípios que serão utilizados em novas experiências, diferentes em termos de tempo e lugar (FONSECA; CUNHA, 2003; FONSECA; CUNHA; ENUMO, 2002; TZURIEL, 1999, 2001).

A EAM é o principal conceito da Teoria da Experiência de Aprendizagem Mediada, Mediated Learning Experience - MLE theory. Essa teoria faz parte da abordagem da Modificabilidade Cognitiva Estrutural (Structural Cognitive Modifiability - SCM - Theory), baseada na principal premissa de que o desenvolvimento cognitivo de qualquer indivíduo é estruturalmente modificável. A teoria da Modificabilidade Cognitiva Estrutural (MCE) se centra no postulado de que é possível, a partir de um enriquecimento interativo, o adulto modificar cognitivamente a criança e reduzir a discrepância entre o desempenho típico e o desempenho potencial do desenvolvimento cognitivo infantil (FEUERSTEIN; FEUERSTEIN, 1991; FONSECA, 2002; KLEIN, 1996; LIDZ, 1991, 2000, 2002; TZURIEL, 1999, 2001).

A EAM representa a qualidade da interação, a qual dependerá de todo o contexto da interação, incluindo todos os elementos presentes: o estímulo, o mediado (a criança), e, principalmente, o mediador (a mãe). Assim, na interação mãe-criança, a EAM pode ser compreendida como um processo mediacional em via-de-mão-dupla interativa, em que a mãe envolve a criança em uma série de 
atividades e processos de resolução de problemas, com dois objetivos principais: (a) auxiliar a criança a enfrentar com eficiência as tarefas ou problemas, e (b) enriquecer o repertório de comportamentos de adaptação da criança, tornandoa autônoma em atividades futuras.

Existem evidências de que é estreita a relação entre aprendizagem mediada, interação entre a criança e a família, e modificabilidade cognitiva (TZURIEL, 1999). Na perspectiva da EAM, o adulto (mãe ou outro) modifica um estímulo pela freqüência, ordem, intensidade e contexto, o que provoca na criança sua curiosidade e capacidade perceptiva; favorecendo, assim, o progresso de suas funções cognitivas. Logo, as interações de aprendizagem mediada no contexto da interação mãe-criança favorecem o desenvolvimento de várias funções cognitivas, beneficiando a aprendizagem da criança em diferentes contextos.

Com relação aos princípios de uma aprendizagem mediada, segundo Feuerstein e Feurstein (1991) e Tzuriel (1999), existem três fatores principais para que uma interação possa ser considerada uma EAM: a intencionalidade e reciprocidade, a transcendência e a significação. A intencionalidade e reciprocidade ocorrem quando um mediador intencionalmente chama a atenção da criança para um objeto e ela responde a este estímulo. Importante considerar que a intencionalidade e reciprocidade são elementos fundamentais no processo de aprendizagem mediada, uma vez que criam na criança um estado de alerta e facilitam o registro eficiente de informações (fase de input), um processamento adequado (fase de elaboração) e uma resposta eficiente (fase de output).

A transcendência diz respeito à atitude do mediador de conduzir a criança para além de contextos concretos ou de necessidades imediatas, de forma que ela aprenda princípios gerais e objetivos que não se limitem ao contexto do "aqui e agora" ou de uma situação ou contexto específico. Em uma situação de interação mãe-criança, o adulto deve, através da observação desse aspecto, favorecer a generalização da aprendizagem de regras, estratégias e princípios de uma situação de experiência concreta para situações novas.

Por fim, a significação se refere ao comportamento do mediador em enfatizar a importância de um estímulo, por meio da expressão de afeto e pela indicação do valor e significado do mesmo. De acordo com este princípio, a criança aprende o significado dos estímulos em uma situação de aprendizagem mediada e internaliza este processo, passando, mais tarde, espontaneamente, a buscar o significado de novas informações, sem ficar passivamente esperando por ajuda do outro.

Além desses componentes, existem outras características importantes que devem estar presentes no comportamento do mediador, como a mediação de: sentimentos de competência, da regulação e controle do comportamento da criança; do comportamento de partilhar; da diferenciação individual e psicológica; dos objetivos de busca, setting, planejamento, realização e desafio; de mudança; e, por fim, a mediação de alternativa otimizada (FEUERSTEIN et al. 1987; LIDZ, 1991). 
Apesar dos três primeiros critérios de mediação - intencionalidade/ reciprocidade, transcendência e significação - serem considerados indispensáveis para uma interação se tornar uma EAM, todos esses componentes ou características do comportamento do mediador são extremamente importantes em toda e qualquer interação baseada em estratégias de aprendizagem mediada.

\section{RELAÇÃO ENTRE EXPERIÊNCIA DE APRENDIZAGEM MEDIADA (EAM), INTERAÇÃO MÃE-CRIANÇA E DESENVOLVIMENTO COGNITIVO INFANTIL}

Diferentes estudos revelam que as crianças cujas mães apresentam altos níveis de mediação internalizam esse mecanismo de mediação e mostram melhor desempenho em situações de avaliação de habilidades cognitivas ou contextos específicos de aprendizagem (TZURIEL, 1999; TZURIEL; ERAN, 1990; TZURIEL; WEISS, 1998). Nesses estudos, segundo análise de Tzuriel (1999), o princípio da mediação por transcendência revelou-se o mais poderoso preditor de mudança no processamento cognitivo da criança. Isso equivale dizer que a mediação que ocorre na interação mãe-criança é internalizada como uma estratégia a ser usada pela criança em outros contextos de aprendizagem.

Ao contrário, um padrão inadequado de mediação materna, decorrente de baixas expectativas das mães acerca da capacidade de aprendizagem infantil ou de uma postura materna pouco ativa, mais diretiva e controladora, pode afetar o desenvolvimento cognitivo infantil, sobretudo quando a criança tem NEE.

Tomando a deficiência visual como exemplo, observa-se um padrão de interação, com baixos níveis de comportamentos maternos mediadores, pode ser provocado pela compreensão inadequada do que é a deficiência visual do filho, ou melhor, dos reais limites e possibilidades de aprendizagem da criança. Dessa forma, as mães de crianças com DV podem não desenvolver a prática de incentivar seu filho a explorar o ambiente, limitando a possibilidade de estabelecer com ele uma interação rica em EAM, que tenha como objetivo principal afetar a flexibilidade e plasticidade mental da criança.

O efeito da deficiência visual sobre a dinâmica familiar atinge diferentes aspectos da interação da família com a criança. Muitos pais de crianças com DV duvidam da sua capacidade em administrar aspectos práticos e emocionais na criação de seus filhos, sentindo-se impotentes diante de situações da vida diária e intimidados ou inadequados para cuidar de uma criança que não enxerga (BOLINGER; BOLINGER, 1996). Embora esses sentimentos possam ser considerados normais, para esses autores, tais sentimentos diminuem a percepção dos pais sobre como manejar as situações de interação com a criança com DV.

Analisando brevemente o processo de desenvolvimento da criança com DV, a restrição de habilidades de exploração, assim como as influências psicológicas da própria criança e do seu ambiente, começa desde o nascimento a 
moldar o seu desenvolvimento. No que se refere ao desenvolvimento cognitivo, uma das principais dificuldades que a criança com DV enfrenta diz respeito à lacuna na apreensão dos estímulos, devido à ausência da percepção visual. É sabido que a percepção visual é um dos principais canais de veiculação e acesso às informações que serão, posteriormente, utilizadas para construção das representações mentais da criança sobre o mundo. Logo, o papel que a visão desempenha como organizadora dos eventos e integradora das informações do ambiente para a criança normal não tem correlato na criança com DV (RECCHIA, 1977).

Algumas vezes, a criança com DV pode apresentar problemas em várias áreas do desenvolvimento cognitivo, incluindo a categorização, a noção de objeto permanente e a aprendizagem (BIGELOW, 1990). A atividade de categorização, por exemplo, é mais difícil para a criança com DV do que para a criança com visão normal. Nesta última, a aprendizagem de categorias se inicia pela escolha e ordenação de objetos e pessoas pelos seus atributos físicos (forma e cor), para posterior ordenação dos mesmos em termos de grupos, função e associação. Para a criança com DV, em função de não poder alcançar as semelhanças e diferenças dos objetos através do canal visual, ela deverá aprender a categorizar pela indicação verbal das semelhanças ou pela percepção táctil. Nesse sentido, o outro terá papel fundamental, já que a criança com DV dependerá de uma outra pessoa para chamar sua atenção para os atributos principais que diferenciam um objeto do outro.

Em relação ao desenvolvimento cognitivo, é necessário oferecer à criança com DV, sistematicamente e de forma planejada, experiências de aprendizagem que a criança normal tem de modo assistemático e não planejado, quando ela interage em um ambiente rico em experiências (BATISTA; ENUMO, 2000). Essas experiências de aprendizagem a serem oferecidas à criança com DV devem ter como princípio uma mediação de qualidade que favoreça o desenvolvimento do pleno potencial de aprendizagem.

\section{A Escala de Avaliação da Experiência de Aprendizagem Mediada (Mediated LEARNING EXPERIENCE -MLE- RATE SCALE: UMA BREVE DESCRIÇÃO}

De acordo com Ferrel (1996), para toda e qualquer criança com necessidades especiais, inclusive com DV, o diferencial no desenvolvimento cognitivo infantil reside em identificar como a criança irá aprender e não o que a criança é capaz de aprender cognitivamente.

No entanto, como saber qual a quantidade e a qualidade de situações de EAM necessárias para alcançar o objetivo acima? E como intervir na interação da mãe com a criança, com objetivo de promover situações de EAM e melhorar o padrão de mediação materna? 
Para discutir essas questões, o presente artigo apresenta uma proposta de operacionalização da Mediated Learning Experience (MLE) Rate Scale (Escala de Avaliação da Experiência de Aprendizagem Mediada), elaborada por Lidz (1991).

A Escala MLE pode ser reconhecida como um instrumento que oferece uma avaliação do repertório de comportamentos do mediador em áreas que são relevantes para o desenvolvimento cognitivo da criança. Foi proposta por Lidz (1991) como uma tentativa de calcular as experiências ambientais e de socialização que têm potencial influência no desenvolvimento cognitivo da criança e de operacionalizar os critérios de EAM decorrentes dos trabalhos clínicos de Reuven Feuerstein e colaboradores.

A Escala MLE inclui a maior parte dos componentes descritos por Feuerstein, além de modificações e inovações decorrentes da literatura sobre pesquisas acerca da interação pais-criança. Inclui 12 componentes do comportamento do mediador que são analisados em termos de potenciais atitudes de mediação de aprendizagem: intencionalidade (intentionality), significação (meaning), transcendência (trancendence), atenção partilhada (joint regard), experiência partilhada sharing experiences), regulação na tarefa (task regulation), elogiar (praise-encouragement), desafiar (challenge), diferenciação psicológica (psychological differentiation), responsividade contingente (contingent responsivity), envolvimento afetivo (affective involvement) e mudança (change).

Cabe ressaltar que o último critério de mediação, mudança, não é utilizado para analisar o padrão de mediação materno, uma vez que, segundo Lidz (1991), esse critério de mediação não é observado no caso da interação mãecriança. Na Escala MLE, é incluído ainda um componente para análise do comportamento da criança na interação com o mediador: a reciprocidade da criança (reciprocity). Este componente se refere ao nível de receptividade da criança às iniciativas de mediação do adulto, ou seja, o quanto a criança está receptiva aos estímulos (comportamentos de mediação) do mediador na interação.

Quanto à análise dos resultados da escala, cada um dos componentes da Escala MLE é avaliado em uma escala de quatro níveis, com um escore total máximo possível de 27 pontos, que variam do nível 0 (ausência de mediação) até o nível 3 (nível máximo ou nível ótimo de mediação). O nível "ótimo" de mediação diz respeito aos resultados esperados de um funcionamento cognitivo apropriado: aprendizagem ativa, auto-regulação, estratégias de resolução de problemas e pensamento representacional. Esses níveis são determinados a partir das atitudes adequadas do mediador em proporcionar a criança situações de interação baseadas em EAM (LIDZ, 1991).

A Escala MLE pode ser indicada como sendo um instrumento adequado para avaliação da mediação materna e já foi utilizada em outros estudos, tendo sido considerada de aplicação mais fácil quando comparada com outros instrumentos de avaliação de EAM, além de requerer um período menor 
de treinamento, como ocorre tipicamente nessa área (LIDZ, 1991, 2002; LIDZ; BOND; DISSINGER, 1990; ZAMBRANA-ORTIZ; LIDZ, 1995).

Com relação à análise das propriedades da Escala MLE, ZambranaOrtiz e Lidz (1995) encontraram forte evidência de confiabilidade e de consistência interna, superior ao nível recomendado de $90 \%$, como encontrado em estudo anterior de Lidz, Bond e Dissinger (1990). Logo, a Escala MLE é um instrumento confiável com relação à consistência interna e fornece uma ampla análise qualitativa, em que é avaliada uma variedade de aspectos da mediação presente na interação adulto-criança.

Utilizada em diferentes estudos sobre interação adulto-criança, a Escala MLE já foi traduzida para o alemão e o espanhol, e tem sido modificada em diferentes versões para aplicação específica em intervenções com pais, professores e trabalhos de desenvolvimento de habilidades de leitura (ZAMBRANA-ORTIZ; LIDZ, 1995). A presente versão em português serviu de base para esta proposta de operacionalização da Escala MLE utilizada em pesquisa com crianças com deficiência visual, sendo detalhadamente apresentada neste artigo.

\section{Método \\ Participantes}

A amostra estudada incluiu 12 díades mãe-criança com deficiência visual que freqüentavam instituições especializadas de atendimento e ensino, localizadas nos municípios de duas capitais da região sudeste do país. As mães tinham entre 30 e 60 anos (média $=40$ anos); a maioria trabalhava em casa (5) ou exerciam atividades remuneradas sem vínculo empregatício (4), apenas 3 trabalhavam fora do lar. A idade das crianças variou de 5 anos e 2 meses a 9 anos, com média de 7 anos e 6 meses de idade, sendo 4 meninos e 8 meninas. Todas as crianças tinham deficiência visual, com predomínio de baixa visão leve, segundo avaliação do oftalmologista responsável pelo tratamento e acompanhamento médico da criança. Todas freqüentavam a escola, a maioria $(n=11)$ em classe regular e 8 delas ainda recebiam atendimento especializado em instituições de Educação Especial e Reabilitação.

\section{INSTRUMENTOS E MATERIAIS}

Para operacionalização da Mediated Learning Experience (MLE) Rate Scale - Escala de Avaliação da Experiência de Aprendizagem Mediada (LIDZ, 1991), foi utilizada uma tradução livre da Escala MLE original, realizada pela primeira autora deste trabalho, com autorização da autora da escala, para uso em pesquisa.

Igualmente, para ser usado na operacionalização da Escala MLE, foi criado um sistema de categorias de comportamentos mediadores que incluiu 36 categorias de análise do comportamento mediador, como, por exemplo, solicitar 
atenção e reflexão da criança, oferecer feedback informativo, positivo e corretivo, explicitar o objetivo da interação, apresentar dicas, modelos e exemplos, demonstrar afeto, encorajar a iniciativa da criança etc.

Três tipos diferentes de jogos de dominó com níveis crescentes de dificuldade conceitual, de acordo com o número de categorias de classificação (A1frutas, A2-frutas e animais e A3- frutas, animais e objetos) foram usados como materiais para que as mães interagissem com as crianças na situação semiestruturada em que foi aplicada a versão operacionalizada da Escala MLE. Os jogos de dominó A1 e A2 continham, ao todo, 21 peças, enquanto que o jogo A3 continha 28 peças. Cabe esclarecer que a dificuldade dos jogos de dominó residia na inclusão de mais uma categoria de classificação além da categoria presente no jogo anterior, sendo que, para o último jogo, foi também aumentado o número de peças.

Os jogos de dominó foram elaborados especialmente para o estudo, com o objetivo de prover um material lúdico que facilitasse a mediação da mãe e fosse adequado à condição visual da criança, já que as peças do dominó foram confeccionadas em madeira com dimensões maiores do que as comuns $(11,5 \mathrm{~cm} X$ $5,5 \mathrm{~cm} \mathrm{X} 1 \mathrm{~cm}$ ) e com figuras coloridas em relevo em material emborrachado, para facilitar o reconhecimento, também através do tato, para as crianças com maior comprometimento visual.

\section{Procedimento}

Cada díade mãe-criança foi filmada em três sessões, cada uma com duração entre 3 minutos e 15 segundos até 15 minutos e 43 segundos, interagindo com o filho em situação semi-estruturada de jogo de dominó com três níveis crescentes de dificuldade (A1, A2, A3), no laboratório das universidades, totalizando 36 sessões de gravação, ao longo de duas semanas.

Para análise do padrão de mediação da mãe na interação com a criança, foi utilizada a versão operacionalizada da Escala MLE. Na operacionalização da Escala MLE, os níveis de mediação para cada um dos 12 componentes da Escala MLE foram definidos com base no sistema de comportamentos mediadores, estabelecidos em duas etapas, descritas a seguir.

Na primeira etapa, tomou-se como referência os pressupostos teóricos da abordagem da MCE e da EAM, um sistema de categorias de comportamentos interativos do mediador com crianças com DV em situação de avaliação assistida (FONSECA; CUNHA; ENUMO, 2002), e estudos sobre interação mãe-criança em situação de jogo, os quais serviram de base para elaboração do sistema de categorias de comportamentos mediadores. Após a tradução livre da Escala MLE, foi criado o sistema de categorias de comportamentos mediadores, para ser usado na análise do padrão de mediação adotado pela mãe na interação com a criança com DV em situação de jogo de dominó. 
$\mathrm{Na}$ segunda etapa, utilizando-se o sistema de categorias de comportamentos mediadores, foram definidos os quatro níveis de mediação para cada um dos 12 componentes da escala. Os níveis de mediação seguiam um gradiente que variava do nível 0 (ausência de mediação) até o nível 3 (nível ótimo de mediação), como na versão original da Escala MLE. Admitia-se a presença de um comportamento mediador em mais de um nível de mediação, como, por exemplo, para o critério de mediação significação, em que, para o nível 1, foi definida a categoria dar feedback informativo e, para o nível 2, além da categoria anterior (dar feedback informativo), foi incluída a categoria enfatizar a relevância de aspectos da interação.

Da mesma forma, admitiu-se a presença de um comportamento mediador em mais de um componente da Escala, como, por exemplo, os critérios de mediação desafiar e diferenciação psicológica, em que a categoria encorajar a iniciativa da criança aparece nos níveis 2 e 3 de ambos os critérios; assim como as categorias oferecer desafios (nível 2) e solicitar reflexão (nível 3), para o critério desafiar; e as categorias ser diretivo (nível 2) e ter au to-regulação (nível 3), para o critério diferenciação psicológica.

Na aplicação da versão operacionalizada da Escala MLE, o nível de mediação materno foi computado pela freqüência dos comportamentos mediadores para cada um dos componentes da Escala, de acordo com a seguinte classificação:

Nível 0: quando a mãe somente apresentava comportamentos verbais e não-verbais que não se relacionavam com a proposta da interação, ou seja, ela demonstrava interesse em aspectos da situação que não se referiam à atividade (jogo de dominó);

Nível 1: quando a mãe apresentava comportamentos verbais e nãoverbais que se relacionavam com a proposta da interação, mas não funcionavam como mediadores da aprendizagem da criança na atividade;

Nível 2: quando a mãe apresentava, de forma assistemática, comportamentos verbais e não-verbais que se relacionavam com a proposta da interação e que tinham potencial de mediar a aprendizagem da criança na atividade;

Nível 3; quando a mãe apresentava, de forma sistemática, comportamentos verbais e não-verbais que se relacionavam com a proposta da interação e que tinham potencial de mediar a aprendizagem da criança, demonstrando claro objetivo de estimular a participação da criança na atividade.

Resumindo, a operacionalização da Escala MLE resultou em um sistema de categorias de comportamentos mediadores que incluiu 36 categorias de análise, organizadas nos 4 níveis de mediação para cada um dos 12 componentes ou critérios de mediação em Experiência de Aprendizagem Mediada (EAM), propostos na versão original da Mediated Learning Experience Rate Scale de Lidz (1991), como pode ser melhor compreendido pela análise do APÊNDICE A. 


\section{Processamento e ANÁlise de Dados}

A partir da análise dos vídeos e utilizando-se um protocolo de registro do comportamento materno em situação de jogo de dominó, foi registrada a freqüência dos comportamentos mediadores das mães. A partir desses registros, foram calculados os níveis de mediação para cada componente/critério de mediação da Escala MLE. Ainda, para cada mãe, foi calculada a média geral dos níveis de mediação obtida pela soma de todos os níveis de mediação para os diferentes componentes da Escala MLE, no total, e por sessão de jogo de dominó.

Com objetivo de garantir a fidedignidade da avaliação do padrão de mediação materno, foi calculado o índice de concordância das classificações em termos dos níveis de mediação obtido em cada componente/critério de mediação da Escala MLE nos jogos de dominó. Para tanto, foram selecionados, aleatoriamente, os protocolos de registro da mediação materna para $25 \%$ da amostra total de mães $(n=3)$. Uma bolsista de iniciação científica (CNPq), estudante de Psicologia, atuou como um segundo juiz. Cabe ressaltar que não foram obtidos índices de concordância menores que $70 \%(72,7 \%$ a $81,8 \%)$.

\section{Resultados}

Os dados dos comportamentos das mães resultantes da aplicação da versão operacionalizada da Escala MLE foram analisados em termos do padrão de interação materno com a criança na situação de jogos de dominó. O padrão de interação foi identificado pela freqüência de comportamentos de mediação, que resultou no nível de mediação adotado pela mãe na interação com a criança, de acordo com os critérios de mediação em experiência de aprendizagem mediada (EAM). Na Tabela 1, estão as medianas e amplitudes de variação dos níveis médios de mediação das mães para o total de critérios de mediação em EAM.

Observa-se que, no Jogo A1, o nível mais alto de mediação, nível 3, foi apresentado por cinco mães: M1, M2, M4, M7 e M8. Dessas mães, apenas uma (M8) manteve um padrão de mediação ótimo (nível3) nos dois jogos subseqüentes. As demais mães diminuíram o nível de mediação do Jogo A1 para A2, sendo que três dessas mães voltaram a elevar seu padrão de mediação (M1, M2 e M7) e uma mãe (M4) manteve baixo o seu padrão de mediação no Jogo A3.

O nível 2 durante o jogo A1 foi observado para duas mães: M5 e M9; sendo que, para M5, o nível médio de mediação diminuiu consideravelmente nos jogos subseqüentes, atingindo o nível 0 e, para M9, o nível 2 se manteve ao longo dos jogos. Semelhante ocorreu para o nível 1: duas mães (M6 e M11) apresentaram este nível no Jogo A1, sendo que uma mãe (M6) manteve este nível ao longo dos jogos e a outra mãe (M11) elevou para nível 2 seu padrão de mediação no Jogo A2, voltando a diminuir o padrão de mediação para o nível 1 no jogo A3. 
CUNHA, A. C. B.; CANAL, C. P. P.; ENUMO, S. R. F.

Tabela 1 - Níveis de mediação materna em cada jogo de dominó ( $\mathrm{n}=12)$

\begin{tabular}{llllll}
\hline $\begin{array}{l}\text { Jogos } \\
\text { Mães }\end{array}$ & $A l$ & $A 2$ & $A 3$ & $M d$ & $A V$ \\
\hline M1-Fernanda & 3 & 1 & 3 & 3 & $1-3$ \\
M2-Lucélia & 3 & 2 & 3 & 3 & $2-3$ \\
M3-Edith & 0 & 2 & 0 & 0 & $0-2$ \\
M4-Paula & 3 & 1 & 1 & 1 & $1-3$ \\
M5-Greice & 2 & 0 & 0 & 0 & $0-2$ \\
M6-Leda & 1 & 1 & 1 & 1 & $1-1$ \\
M7- Pilar & 3 & 1 & 3 & 3 & $1-3$ \\
M8- Márcia & 3 & 3 & 3 & 3 & $2-3$ \\
M9- Zuleica & 2 & 2 & 2 & 2 & $2-2$ \\
M10- Jane & 0 & 1 & 1 & 1 & $0-1$ \\
M11- Rute & 1 & 2 & 1 & 1 & $1-2$ \\
M12- Cristiane & 0 & 0 & 1 & 0 & $0-1$ \\
\hline Md & 3 & 1 & 1 & 3 & $0-3$ \\
\hline
\end{tabular}

$\mathrm{Md}=$ mediana; $\mathrm{AV}=$ amplitude de variação.

Foi observada ainda a ocorrência do nível 0 , ou seja, ausência de mediação durante a interação, o que ocorreu para três mães (M3, M10 e M12). Das três mães que apresentaram nível 0 no Jogo A1, uma mãe (M12) manteve este nível no Jogo A2, passando a apresentar nível 1 no Jogo A3. As mães M3 e M10 apresentaram desempenho bem variado; enquanto que o padrão de mediação da M3 se elevou para nível 2 no Jogo A2, voltando a apresentar nível 0 no Jogo A3; o padrão de mediação da M10 se elevou para nível 1 no Jogo A2, mantendo este nível também no Jogo A3.

Em resumo, pode-se observar pelo exame da Tabela 1 que o nível de mediação materno variou ao longo dos diferentes jogos de dominó. No jogo A1, o nível mais freqüente foi o nível 3, enquanto que no Jogo A2 o nível mais freqüente foi o nível 1, o que se repetiu no Jogo A3.

A Figura 1 apresenta o padrão de mediação das mães, analisado de acordo com as medianas do nível médio de mediação, para cada critério de mediação avaliado pela Escala EAM, no total dos três jogos de dominó (A1, A2 e A3). 


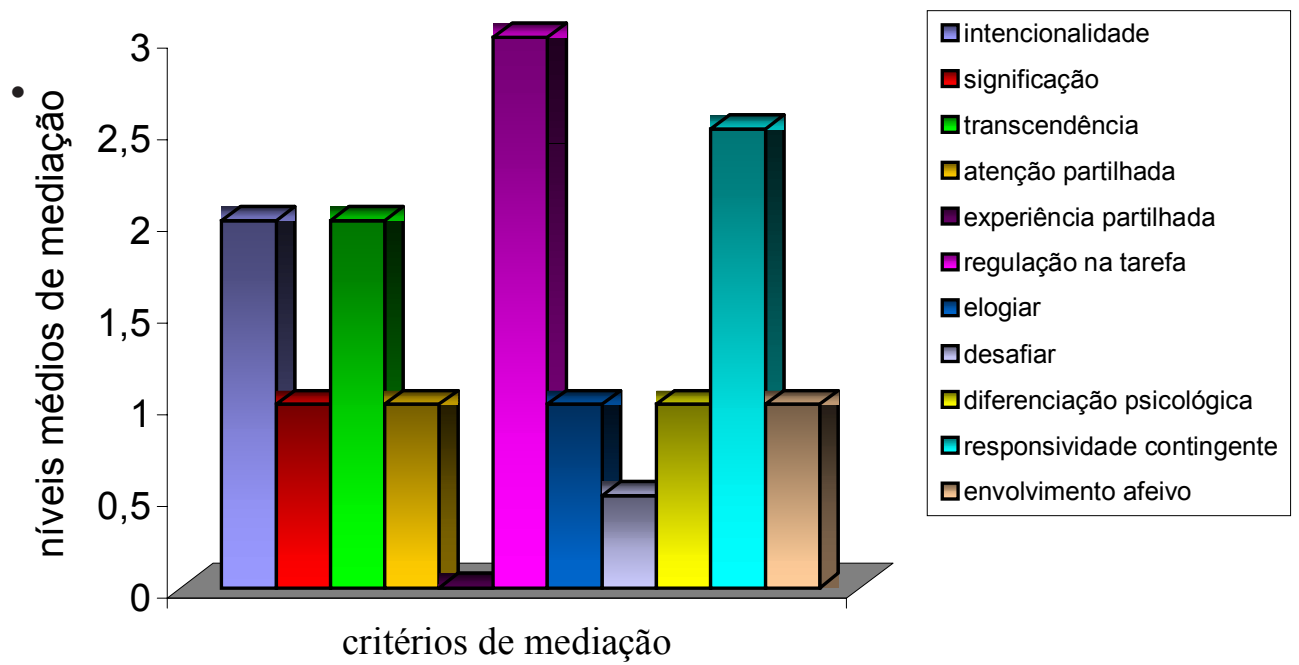

Figura 1 - Níveis de mediação materna nos três jogos de dominó, em cada critério de Experiência de Aprendizagem Mediada (EAM) $(n=12)$.

Observa-se, na Figura 1, que, nos critérios regulação na tarefa $(\mathrm{Md}=3)$ e responsividade contingente ( $\mathrm{Md}=2,5)$, as mães adotaram o maior nível de mediação; isto equivale dizer que as mães apresentaram um ótimo nível de mediação, no que se refere às habilidades de responder sempre às iniciativas de interação da criança e regular o comportamento dela na execução da tarefa pela apresentação freqüente de comportamentos de mandar, oferecer dicas e feedback corretivos e fazer perguntas, com objetivo de facilitar o desempenho da criança no jogo.

Para intencionalidade e transcendência, o nível médio de mediação (Md = 2) foi o mais adotado pelas mães. O padrão de mediação materna ser classificado nesse nível, no que se refere à intencionalidade, significa que a mãe apresentou para a criança, durante os jogos de dominó, comportamentos de solicitar a atenção e explicitar o objetivo da interação, sem, no entanto, solicitar reflexão da criança, o que faria com que a mãe tivesse classificação em nível máximo (3) para esta categoria.

Já para o critério de mediação transcendência, as mães obtiveram nível 2 porque, para auxiliar a criança no jogo de dominó, evocaram experiências e ofereceram modelos relacionados à interação sem fazer uso de pontes cognitivas conceituais e temporais, para facilitar o desempenho da criança no jogo, o que equivaleria ao nível 3. Quando a mãe, além de comentar o foco de interesse da criança, estabelecia claramente uma atenção conjunta com ela e demonstrava ser empática, ou seja, verbalizava sobre possíveis pensamentos e sentimentos da criança relacionados à atividade, ela adotava o nível 2 de mediação em atenção partilhada. 
O nível 1 de mediação, nível mais elementar, que ocorria quando a mãe apresentava comportamentos verbais e não-verbais que se relacionavam com a proposta da interação, mas sem intenção explícita de mediar a aprendizagem da criança na atividade, apareceu como mais freqüente em 5 categorias de mediação: significação, atenção partilhada, elogiar, diferenciação psicológica e envolvimento afetivo.

Para categoria significação, o nível 1 quer dizer que a mãe ofereceu informações sobre os aspectos do jogo, sem enfatizar a relevância desses aspectos e/ou expandir o significado dessas informações. No caso de atenção partilhada, a mãe dirigia-se à criança, sem encorajar sua iniciativa e/ou comentar o seu foco de atenção. Para o critério de mediação elogiar, o nível 1 significava que a mãe elogiava a criança sem encorajar a sua iniciativa ou mesmo comentar seu desempenho durante a partida de dominó. Em relação à diferenciação psicológica, a mãe apresentava comportamentos verbais e não-verbais de auto-regulação, incentivando a iniciativa da criança em 1 ou 2 situações de interação, porém direcionando a execução da atividade na maioria das situações. Por fim, o nível 1 para envolvimento afetivo quer dizer que a mãe demonstrou afeto na interação com a criança em 1 ou 2 situações apenas.

Para o critério desafiar, a mediana do nível médio de mediação foi 0,5, enquanto que a mediana para experiência partilhada foi 0 . Cabe destacar que, quando se realiza a análise individualizada do padrão de interação materno, isto não significa a ausência de mediação em relação, pelo menos, ao critério desafiar, ao longo dos jogos de dominó. já que para esse critério de mediação, duas mães obtiveram nível 1.

\section{Discussão}

A proposta de operacionalizar a Escala MLE para este estudo teve como objetivo adequá-la à situação específica de investigação. Seguindo as mesmas definições dos componentes e do gradiente de mediação da versão original da Escala, foi elaborado um sistema de categorias de comportamentos mediadores, com categorias de análise, orientadas para a avaliação da interação mãe-criança com DV, na situação semi-estruturada da pesquisa (jogo de dominó). definido como:

Por exemplo, o critério transcendência, na versão original da Escala, é

[...] uma atitude do mediador de estabelecer uma ponte cognitiva entre a tarefa ou atividade e as experiências relacionadas, mas não atuais, da criança, referindose ao passado ou antecipando o futuro. Inclui atitude do mediador em estabelecer uma ponte cognitiva que promova imagens visuais e ajude a criança a mudar de uma resposta perceptual para uma conceitual (LIDZ, 1991, p. 107).

$\mathrm{Na}$ versão operacionalizada, foram incluídas categorias de comportamento materno, como: comentar descritivamente, evocar experiências, oferecer modelos e estabelecer ponte cognitiva conceitual e temporal, com exemplos de situações específicas da interação da mãe com a criança no jogo de dominó. 
Como dito anteriormente, este sistema de categorias de comportamentos mediadores foi elaborado a partir da análise de observações em vídeo da interação mediador-criança com DV, através de discussões em grupo de pesquisa com base nos pressupostos teóricos da abordagem da teoria da Experiência de Aprendizagem Mediada. Esse procedimento teve como base um conjunto preciso de hipóteses e um amplo processo de trocas com o material, o que, para Kreppner (2001), são medidas indispensáveis para elaboração de categorias de análise da interação social. Para esse autor, antes que as abstrações e categorias sejam formuladas, deve-se ter claro o problema e os objetivos da pesquisa, assim como uma apreciação extensiva do material de análise, sem os quais nenhuma categoria pode ser formulada.

Além disso, já que a interação da díade no jogo de dominó foi registrada em vídeo, a escolha das categorias de análise pôde ser postergada e adaptada às condições específicas encontradas durante as próprias observações e moldada de acordo com as especificidades da situação de interação, uma vez que a interação da díade pôde ser observada repetidamente, comparando-se as situações observadas (KREPPNER, 2001).

Nesse sentido, considera-se que esta proposta de operacionalização da Escala MLE, contendo um sistema de categorias de comportamentos mediadores, pode ser indicada como um instrumento adequado para análise, tanto quantitativa quanto qualitativa, da interação entre o adulto e a criança em situação semi-estruturada de aprendizagem, o que foi corroborado pela obtenção de índices de concordância aceitáveis.

Por fim, cabe concluir que, partindo do princípio de que, na interação com a criança, o adulto, quer seja ele a mãe, o professor ou até o terapeuta, pode apresentar baixas expectativas com relação ao desenvolvimento infantil, passando, assim, a adotar um padrão inadequado de mediação na interação com a criança. A utilização da presente proposta de operacionalização da Escala MLE pode servir de base para elaboração de programas de orientação e capacitação de pais e professores que tenham como objetivo favorecer a construção de uma visão mais positiva e mais otimista acerca do potencial de aprendizagem infantil, com conseqüente mudança na postura do adulto na interação com a criança com necessidades especiais.

Com efeito, a Escala MLE operacionalizada pode oferecer diretrizes claras, concretas e específicas para auxiliar na recomendação e elaboração de planos de intervenção baseados na observação da interação entre o adulto e a criança. Igualmente, pode ajudar no monitoramento da evolução do comportamento do mediador em programas de intervenção, além de ajudar a descrever os componentes interacionais nos diferentes contextos onde a criança interage, sobretudo no contexto da interação mãe-criança. 


\section{REFERÊNCIAS}

BATISTA, C. G.; ENUMO, S. R. F. Desenvolvimento humano eimpedimentos de origem orgânica: o caso da deficiência visual. In: NOVO, H. A.; MENANDRO, M. C. S.(Orgs.). Olhares diversos: estudando o desenvolvimento humano. Vitória, ES: CAPES/PROIN, Programa de PósGraduação em Psicologia/Universidade Federal do Espírito Santo, 2000. p. 157-174.

BIGELOW, A. Relationship between the development of language and though in young blind children. Journal of Visual Impairment \& Blindness, v. 84, n. 10, p. 414-419, 1990.

BOLINGER, R.; BOLINGER, C. Family Life. In: HOLBROOK, M.C. (Ed.). Children with visual impairments: a parents' guide. The Special-Needs Collection. EUA: Woodbine House, 1996. p. 129-157.

CUNHA, A. C. B.; ENUMO, S. R. F.; PEDROZA, C. P. Será que o meu filho tem potencial de aprendizagem: breve análise da relação cognitiva dinâmica da criança com deficiência visual (D.V.) e interacção mãe-criança. Integrar, Lisboa, v. 21-22, p. 47-52, 2004.

FERREL, K. A. Your child's development. In: HOLBROOK, M. C. (Ed.). Children with visual impairments: a parents' guide. New York: Woodbine House, 1996. p. 73-96.

FEUERSTEIN, R.; FEUERSTEIN, S. Mediated learning experience: a theorical review. In: FEUERSTEIN, R.; KLEIN, P. S.; TANNEMBAUM, A. J. (Eds.). Mediated Learning Experience (MLE): Theorical, psychosocial and learning implications. London: Freund Publishing, 1991. p. 3-51.

FEUERSTEIN, R. et al. Prerequisites for assessment of learning potential: The LPAD model. In: LIDZ, C. S. (Ed.). Dynamic assessment - an interactional approach to evaluating learning potential. New York: Guilford Press, 1987. p. 35-51.

FONSECA, V. Pais e filhos em interação: aprendizagem mediada no contexto familiar. São Paulo: Ed. Salesiano, 2002.

FONSECA, V.; CUNHA, A. C. B. Teoria da Experiência de Aprendizagem Mediatizada e interação familiar: prevenção das perturbações do desenvolvimento e aprendizagem. Lisboa, Portugal: Editora da Faculdade de Motricidade Humana, 2003.

FONSECA, V.; CUNHA, A. C. B.; ENUMO, S. R. F. O desenvolvimento cognitivo da criança com deficiência visual e suas perspectivas de avaliação: da abordagem padronizada à avaliação dinâmica. Revista de Educação Especial e Reabilitação, Lisboa, v. 9, n.1-2, p. 75-91, 2002.

KLEIN, P. S. Early intervention: cross-cultural experiences with mediational approach. New York: Garland Publishing, 1996.

KREPPNER, K. Sobre a maneira de produzir dados. Psicologia: Teoria \& Pesquisa, Brasília, v. 17, n. 2, p. 97-107, 2001.

LIDZ, C. S. Practioner's guide to dynamic assessment. New York: The Guilford Press, 1991.

. Theme and some variations on the concepts of mediated learning experience and dynamic assessment. In: KOZULIN, A.; RAND, Y. (Eds.). Experience of mediated learning: an impact of Feuerstein's theory in Education and Psychology. New York: Pergamon, 2000. p. 166-174.

. Mediated learning experience (MLE) as a basis for an alternative approach to assessment. School Psychology International, v. 23, n.1, p. 68-84, 2002.

LIDZ, C. S.; BOND, L.; DISSINGER, L. Consistency of mother-child interaction using the Mediated Learning Experience (MLE) Scale. Special Services in the Schools, v. 6, n. 1/2, p. 145$165,1990$. 
LINHARES, M. B. M.; MARTINS, I. M. B.; KLEIN, V. C. (2004). Mediação materna como processo de promoção e proteção do desenvolvimento da criança nascida prematura. In: MARTURANO, E. M.; LINHARES, M. B. M.; LOUREIRO, S. R. (Orgs.). Vulnerabilidade e proteção: indicadores na trajetória de desenvolvimento do escolar. São Paulo: Casa do Psicólogo, 2004. p. 39-74

RECCHIA, S. L. Establishing intersubjective experience: developmental challenges for young children with congenital blindness and autism and their caregivers. In: LEWIS, V.; COLLIS. G. M. (Eds.). Blindness and psychological development in young children. Leicester, UK: BPS Books, 1997. p. 116-129.

TZURIEL, D. Parent- child mediated learning interactions as determinants of cognitive modifiability: Recent researchers and future directions. Genetic, Social and General Psychology Monographs, v. 125, n. 2, p. 109-156, 1999.

TZURIEL, D. Dynamic assessment of young children. New York: Kluwer Academic/ Plenum Publishers, 2001.

TZURIEL, D.; ERAN, Z. Inferential cognitive modifiability as a function of mother-child mediated learning experience (MLE) interactions among Kibbutz young children. International Journal of Cognitive Education and Mediated Learning, v. 1, p. 103-117, 1990.

TZURIEL, D.; WEISS, S. Cognitive Modifiability as a function of mother-chid mediated learning strategies, mother's acceptance-rejection, and children's personality. Early Development and Parenting, v. 7, p. 79-99, 1998.

ZAMBRANA-ORTIZ, N. Z.; LIDZ, C. S. The relationship between Puerto Rican mothers' and fathers' mediated learning experiences and the competence of their preschool children. Journal of Cognitive Education, v. 4, n. 1, p. 17-32, 1995. 
APÊNDICE A - Categorias comportamentais do mediador para os componentes ou critérios de mediação da Mediation Learning Experience (MLE) Rate Scale adaptada

\begin{tabular}{|c|c|c|}
\hline $\begin{array}{l}\text { Componente da Escala MLE } \\
\text { (critério de mediação) }\end{array}$ & $\begin{array}{l}\text { Níveis de } \\
\text { mediação } \\
(0-3)\end{array}$ & Categorias de comportamento mediadores \\
\hline \multirow{4}{*}{ INTENCIONALIDADE } & Nível 0 & $\begin{array}{l}\text { ANR: Atuação não relacionada (Ex.: Conversar sobre } \\
\text { eventos de sua vida pessoal). }\end{array}$ \\
\hline & Nível 1 & $\begin{array}{l}\text { AND: Atuação não direcionada (Ex.: Oferecer peça do } \\
\text { jogo para criança e cruzar os braços). }\end{array}$ \\
\hline & Nível 2 & $\begin{array}{l}\text { SOA: Solicitar atenção ( } 1 \text { a } 2 \text { ocorrências). } \\
\text { EOI: Explicitar objetivo da interação ( } 1 \text { a } 2 \\
\text { ocorrências). }\end{array}$ \\
\hline & Nível 3 & $\begin{array}{l}\text { SOA: Solicitar atenção ( } 3 \text { ou mais ocorrências). } \\
\text { EOI: Explicitar objetivo da interação ( } 3 \text { ou mais } \\
\text { ocorrências). } \\
\text { SOR: Solicitar reflexão. }\end{array}$ \\
\hline \multirow{4}{*}{ SIGNIFICAÇÃO } & Nível 0 & ANR: Atuação não relacionada. \\
\hline & Nível 1 & FEI: Dar feedback informativo (1 a 2 ocorrências).. \\
\hline & Nível 2 & $\begin{array}{l}\text { FEI: Dar feedback informativo ( } 3 \text { ou mais ocorrências). } \\
\text { ENF: Enfatizar a relevância de aspectos da interação. }\end{array}$ \\
\hline & Nível 3 & EXP: Expandir significado. \\
\hline \multirow{4}{*}{ TRANSCENDÊNCIA } & Nível 0 & ANR: Atuação não relacionada. \\
\hline & Nível 1 & COA: Comentar descritivamente a atividade. \\
\hline & Nível 2 & $\begin{array}{l}\text { EEX: Evocar experiências (1 a } 2 \text { ocorrências). } \\
\text { MOD: Oferecer modelos ( } 1 \text { a } 2 \text { ocorrências). }\end{array}$ \\
\hline & Nível 3 & $\begin{array}{l}\text { EEX: Evocar experiências ( } 3 \text { ou mais ocorrências). } \\
\text { MOD: Oferecer modelos ( } 3 \text { ou mais ocorrências). } \\
\text { PCT: Fazer Ponte Cognitiva Temporal. } \\
\text { PCC: Fazer Ponte Cognitiva Conceitual. }\end{array}$ \\
\hline \multirow{4}{*}{ ATENÇÃO PARTILHADA } & Nível 0 & ANR: Atuação não relacionada. \\
\hline & Nível 1 & DAC: Dirigir-se à criança. \\
\hline & Nível 2 & $\begin{array}{l}\text { EIC: Encorajar iniciativa da criança ( } 1 \text { a } 2 \text { ocorrências). } \\
\text { CFA: Comentar sobre foco de atenção da criança. } \\
\text { EMP: Ser empático ( } 1 \text { a } 2 \text { ocorrências). }\end{array}$ \\
\hline & Nível 3 & $\begin{array}{l}\text { EMP: Ser empático ( } 3 \text { ou mais ocorrências). } \\
\text { EIC: Encorajar iniciativa da criança ( } 3 \text { ou mais } \\
\text { ocorrências. } \\
\text { EDA: Encorajar demonstração de afeto. }\end{array}$ \\
\hline \multirow{4}{*}{$\begin{array}{l}\text { EXPERIÊNCIA } \\
\text { PARTILHADA }\end{array}$} & Nível 0 & ANR: Atuação não relacionada. \\
\hline & Nível 1 & EXP: Apresentar experiências. \\
\hline & Nível 2 & DEX: Dar exemplos. \\
\hline & Nível 3 & $\begin{array}{l}\text { ERP: Oferecer estratégias de resolução de problema. } \\
\text { PCT: Fazer Ponte Cognitiva Temporal. }\end{array}$ \\
\hline \multirow{4}{*}{ REGULAÇÃO NA TAREFA } & Nível 0 & ANR: Atuação não relacionada. \\
\hline & Nível 1 & MPA: Manipular passivamente. \\
\hline & Nível 2 & $\begin{array}{l}\text { MAT: Manipular ativamente (1 a } 2 \text { ocorrências).. } \\
\text { MAN: Mandar executar. }\end{array}$ \\
\hline & Nível 3 & $\begin{array}{l}\text { MAT: Manipular ativamente ( } 3 \text { ou mais ocorrências).. } \\
\text { FEC: Dar feedback corretivo. } \\
\text { DIC: Oferecer dicas. } \\
\text { PER: Fazer perguntas. }\end{array}$ \\
\hline
\end{tabular}




\begin{tabular}{|c|c|c|}
\hline $\begin{array}{l}\text { Componente da Escala MLE } \\
\text { (critério de mediação) }\end{array}$ & $\begin{array}{l}\text { Níveis de } \\
\text { mediação } \\
\quad(0-3)\end{array}$ & Categorias de comportamento mediadores \\
\hline \multirow{4}{*}{ ELOGIAR } & Nível 0 & ANR: Atuação não relacionada. \\
\hline & Nível 1 & FEP: Dar feedback positivo. \\
\hline & Nível 2 & $\begin{array}{l}\text { FEP: Dar feedback positivo (elogios). } \\
\text { EIC: Encorajar iniciativa da criança. }\end{array}$ \\
\hline & Nível 3 & $\begin{array}{l}\text { FEP: Dar feedback positivo (elogios). } \\
\text { COD: Comentar desempenho da criança. }\end{array}$ \\
\hline \multirow{4}{*}{ DESAFIAR } & Nível 0 & ANR: Atuação não relacionada. \\
\hline & Nível 1 & DES: Oferecer desafios (1 a 2 ocorrências). \\
\hline & Nível 2 & $\begin{array}{l}\text { DES: Oferecer desafios ( } 3 \text { a } 4 \text { ocorrências). } \\
\text { EIC: Encorajar iniciativa da criança. }\end{array}$ \\
\hline & Nível 3 & $\begin{array}{l}\text { DES: Oferecer desafios (mais de } 4 \text { ocorrências). } \\
\text { EIC: Encorajar iniciativa da criança. } \\
\text { SOR: Solicitar reflexão. }\end{array}$ \\
\hline \multirow{4}{*}{$\begin{array}{l}\text { DIFERENCIAÇÃO } \\
\text { PSICOLÓGICA }\end{array}$} & Nível 0 & ANR: Atuação não relacionada. \\
\hline & Nível 1 & DIR: Ser diretivo. \\
\hline & Nível 2 & $\begin{array}{l}\text { DIR: Ser diretivo. } \\
\text { EIC: Encorajar iniciativa da criança. } \\
\text { AUR: Ter auto-regulação. }\end{array}$ \\
\hline & Nível 3 & $\begin{array}{l}\text { EIC: Encorajar iniciativa da criança. } \\
\text { AUR: Ter auto-regulação }\end{array}$ \\
\hline \multirow{4}{*}{$\begin{array}{l}\text { RESPONSIVIDADE } \\
\text { CONTINGENTE }\end{array}$} & Nível 0 & ANC: Atuação não contingente. \\
\hline & Nível 1 & DAC: Dirigir-se à criança (1 a 2 ocorrências). \\
\hline & Nível 2 & $\begin{array}{l}\text { DAC: Dirigir-se à criança ( } 3 \text { ou mais ocorrências). } \\
\text { RAC: Responder à criança ( } 3 \text { ou mais ocorrências). }\end{array}$ \\
\hline & Nível 3 & $\begin{array}{l}\text { DAC: Dirigir-se à criança. } \\
\text { RAC: Responder à criança. (todas as situações de } \\
\text { interação). }\end{array}$ \\
\hline \multirow{4}{*}{$\begin{array}{l}\text { ENVOLVIMENTO } \\
\text { AFETIVO }\end{array}$} & Nível 0 & AIN: Atuação indiferente. \\
\hline & Nível 1 & DEA: Demonstrar Afeto (1 a 2 ocorrências). \\
\hline & Nível 2 & DEA: Demonstrar Afeto (3 a 4 ocorrências). \\
\hline & Nível 3 & $\begin{array}{l}\text { DEA: Demonstrar Afeto (todas as situações de } \\
\text { interação). }\end{array}$ \\
\hline
\end{tabular}

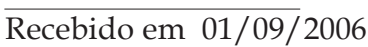

Reformulado em 28/12/2006

Aprovado em 30/12/2006 
CUNHA, A. C. B.; CANAL, C. P. P.; ENUMO, S. R. F. 